\title{
Should the Benefit of Adjuvant Chemotherapy in Colon Cancer Be Re-Evaluated?
}

\author{
Lars A. Påhlman, Uppsala University, Uppsala, Sweden \\ Werner M. Hohenberger and Klaus Matzel, Universitätsklinikums Erlangen, Erlangen, Germany \\ Kenichi Sugihara, Tokyo Medical and Dental University, Tokyo, Japan \\ Philip Quirke, St James's University Hospital, Leeds, United Kingdom \\ Bengt Glimelius, Uppsala University, Uppsala, Sweden
}

Because favorable effects on survival were seen in randomized trials conducted during the 1980s, adjuvant chemotherapy in colon cancer was established as routine therapy in stage III disease in the United States in 1990. ${ }^{1}$ Follow-up trials in the United States, Asia, and Europe $e^{2,3}$ soon meant that it became recommended therapy worldwide, not only in stage III but in stage II disease as well, if risk factors for recurrence were present. Additional trials established the combination of a fluoropyrimidine and oxaliplatin as reference treatment for patients with stage II disease with risk factors who are fit for therapy and for those with stage III disease. ${ }^{4-6}$ The addition of oxaliplatin in the treatment of elderly patients has been questioned. ${ }^{7}$ Here we present arguments questioning not only the addition of oxaliplatin in the treatment of some younger patients as well but also the offering of adjuvant chemotherapy at all to some of these patients.

Medical care continuously develops, and as a consequence, treatment results improve. This development has also been seen in colon (and rectal) cancer, and the improvements actually challenge the established benefit of adjuvant chemotherapy in colon (and rectal) cancer. We question whether the risk of recurrence is sufficiently high for most patients with stage II disease, even when risk factors are present, and for some patients with stage III disease in the presence of high-quality, modern, multidisciplinary team care to motivate adjuvant chemotherapy.

In colorectal cancer, there has been a marked change during the past decades regarding surgery. It started with the total mesorectal excision (TME) technique for rectal cancer. ${ }^{8}$ This technique has now spread around the world; the majority of surgeons have learned how to operate effectively on rectal cancer, and many centers report low local recurrence rates. Population-based data from national quality registers also show that the local recurrence rate can today reach approximately $5 \%,{ }^{9,10}$ equaling the rates achieved in dedicated centers. ${ }^{8,11-13}$ Surgery for colon cancer may also be about to change, with complete mesocolic excision dissection and the concept of central ligation. ${ }^{14}$ These techniques have started to spread among surgeons, and population data already indicate that there may be an overall survival benefit, in addition to improvements reflecting stage migration, if colonic surgery is performed in accordance with such procedures. ${ }^{15-19}$

Preoperative staging of colorectal cancer has also improved, and up-to-date contrast-enhanced computed tomography of the thorax and abdomen, completed with ultrasonography or magnetic resonance imaging with contrast agents in the case of equivocal liver lesions or positron emission tomographycomputed tomography in the case of equivocal findings outside the liver, has also resulted in fewer recurrences in those undergoing surgery (ie, the target patients for adjuvant therapy). The scenario has changed from fewer metachronous to more synchronous metastases. Furthermore, although pathologists cannot reduce recurrence risks per se, better pathologic staging results in lower stage-specific recurrence rates, often referred to as stage migration.

The rectal cancer radiotherapy story illustrates the same kind of problem the medical community is facing when one or several aspects of multidisciplinary care are improved. The story is well known from literature. The reduction in local recurrence rates seen after preoperative radiotherapy ${ }^{20}$ was questioned when surgery was improved (ie, when TME was introduced). The two trials then initiated-the Dutch-Swedish TME trial and the Medical Research Council CR07 trial in the United Kingdom, ${ }^{21,22}$ in which preoperative radiotherapy using the $5 \times 5$ Gy schedule was tested against selective postoperative (chemo)radiotherapy-showed that preoperative radiotherapy significantly reduced local recurrence rates. Actually, the relative reduction may have been slightly larger with TME (hazard ratio, 0.38; absolute difference, 11\% v 4\%) than with the older, suboptimal surgery (hazard ratio, 0.46; absolute difference, $27 \% v 13 \%$ ). The overall survival gain seen previously in the Swedish Rectal Cancer Trial ${ }^{23}$ could not be reproduced; the absolute gain in local recurrences was likely too small to show up in the trials in which TME was used.

The gains from postoperative chemoradiotherapy in local recurrence rates and survival ${ }^{24}$ have not been tested using TME. In a German trial testing pre- versus postoperative chemoradiotherapy, it was shown that preoperative chemoradiotherapy was better than postoperative in reducing the local recurrence rate, approximately $10 \%$ after postoperative treatment versus $7 \%$ after preoperative irradiation. The TME technique was used for most patients in the trial, but no difference in survival could be seen even after long-term follow-up. ${ }^{25}$

On the basis of this knowledge, there is an ongoing debate over whether radiotherapy is needed in the majority of rectal cancers because of the low recurrence rates seen today without 
radiotherapy and the adverse effects of irradiation. The so-called number needed to harm, in addition to the number needed to treat, has become an important issue of discussion. The question to be raised is whether it is necessary to lower the limited risk of local recurrence even further (by approximately 60\%) by adding radiotherapy.

All trials that have tested chemotherapy versus surgery alone were conducted in an era when surgery was far from optimal; classic hemicolectomies were not always performed, and the complete mesocolic excision technique was not used. In addition, preoperative staging and postoperative pathology were suboptimal according to present standards. The recurrence risks in patients undergoing radical surgery (stage I to III disease) are thus likely much lower today than they were when those trials were conducted. Today, many centers can report survival figures clearly better than those seen both with and without chemotherapy in the older trials. In many cancer registries in Europe, patients with stage I or II colon cancer have a 5-year survival rate greater than $90 \%$; for patients with stage III disease, this rate is approximately $80 \%{ }^{14}$ (in Swedish, Danish, and Dutch colorectal cancer registries). The adjuvant chemotherapy administered according to recommendations contributes to these results, and it is impossible to know from modern series what the recurrence risk overall and in each stage would be if no adjuvant chemotherapy were administered. A Nordic group made an attempt to find out the recurrence risk on the basis of a systematic literature review of modern series, where the quality of care (including staging, surgery, and pathology) would be reasonably high. ${ }^{26}$ It was not possible to quantify the risk, but evidence indicated that the risk was considerably lower than in the past, potentially lower than $10 \%$ to $15 \%$ in many groups of patients who would be considered for adjuvant chemotherapy according to most guidelines (eg, those with stage II disease with risk factors like pT4, $<12$ nodes, poor differentiation, or vascular and nerve invasion). There was a clear lack of studies reporting recurrence figures with modern quality of care.

Analogous to the experience in rectal cancer, the relative gains from adjuvant chemotherapy using a fluoropyrimidine alone in colon cancer are likely (at least) to be the same as in the older trials. In stage II disease, these gains range from $20 \%$ to $25 \%$ on the basis of a Cochrane analysis and other systematic overviews. ${ }^{27}$ In stage III disease, they are less well described but anticipated to be higher $(30 \%$ to $35 \%) .^{28}$ Let us assume that the relative gains average $30 \%$ in stage II and III disease combined. If 100 patients are treated with adjuvant fluoropyrimidine alone, and the absolute risk of recurrence is, for example, $20 \%$, six patients will not experience recurrence. Similarly, if the absolute risk of recurrence is $40 \%, 12$ recurrences will be prevented in 100 treated patients. The addition of oxaliplatin further reduces the risk by $18 \%$ to $20 \%$, at least in stage III disease and potentially also in stage II disease with risk factors. Again, if 100 patients are treated, and the absolute risk figures after surgery alone are as we have described (ie, 20\% and $40 \%$, respectively), an additional three (from 20 recurrences down to 14 with a fluoropyrimidine alone and down to approximately 11 with the combination) and six recurrences (from 40 down to 28 and approximately 22), respectively, will be prevented. Today, with optimized staging, surgery, and pathology, if the absolute risks of recurrence after surgery alone in the particular stages of disease are not $20 \%$ and $40 \%$ but instead $10 \%$ and $20 \%$, respectively, the absolute gains are then three and six with a fluoropyrimidine alone and are further reduced by one to two with the addition of oxaliplatin per 100 treated patients. Today, the standard of care at most centers is fluorouracil or capecitabine combined with oxaliplatin (eg, FOLFOX [fluorouracil, leucovorin, and oxaliplatin] or CAPOX [capecitabine plus oxaliplatin] regimen) for all patients with stage III disease and many patients with stage II disease, if risk factors for recurrence are present. Because toxicity resulting from oxaliplatin treatment is not negligible, with sometimes persistent neuropathy, ${ }^{29}$ many patients with a low risk of recurrence will be harmed for little gain.

The present guidelines for adjuvant chemotherapy in stage II colon cancer with and without risk factors and in stage III disease are based on recurrence risks from the past. They are much lower today, although we do not know precisely how much lower. The reasons are mainly better surgery and better preoperative staging, where many patients with metastatic disease have been "converted" to synchronous from metachronous disease. Even if the relative gains from administering adjuvant chemotherapy with or without oxaliplatin are the same as those shown in the trials (with the higher absolute risk figures), the absolute gains are less than they were, and they may be too low to recommend therapy. It is not realistic to conduct a new surgery-alone trial in the groups of greatest interest (ie, those with anticipated recurrence risks of $10 \%$ to $20 \%$, or most patients with stage II and many with stage III disease), as was done for radiotherapy in rectal cancer. Rather, it is important to properly analyze risk figures in large unselected populations where the quality of care is known and with an attempt to control for the effects of adjuvant chemotherapy administered to different proportions of patients. The number needed to treat is high, and thus, many patients will unnecessarily be harmed. Today, we must re-evaluate the need for administering routine adjuvant chemotherapy to many patients with colon cancer when they are receiving high-quality multidisciplinary care. Furthermore, a much more differentiated stratification than simply grouping patients into stages II (with risk factors) and III (A, B, and C) is required; some patients with stage III disease have a much lower risk of recurrence than some patients with stage II. For example (also an example of the amount of information needed in routine care), we do not believe than an otherwise fit 60-year-old patient, who has undergone adequate staging and surgery, with left-sided pT3b, N1 (1 positive, 28 sampled), no vessel (v-) or nerve $\left(\mathrm{N}^{-}\right)$ involvement, and low $\mathrm{V}$ malignancy grade colon cancer has a sufficiently high recurrence risk to recommend adjuvant chemotherapy, certainly not with oxaliplatin. If his or her preoperative carcinoembryonic antigen level is very low or only moderately elevated (eg, < 10) and normalizes postoperatively, the risk of recurrence is likely less than $10 \%$; the absolute benefit of adjuvant combination chemotherapy for this patient is low (four per 100 patients treated) compared with the morbidity associated with receiving the two chemotherapy agents during 6 months (or maybe 3 months in the future). Improving multidisciplinary care to meet modern standards should lead to a reduction in the use of adjuvant therapy in everyday practice.

\section{AUTHORS' DISCLOSURES OF POTENTIAL CONFLICTS OF INTEREST}

Disclosures provided by the authors are available with this article at www.jco.org. 


\section{AUTHOR CONTRIBUTIONS}

Financial support: Bengt Glimelius

Administrative support: Lars A. Påhlman, Bengt Glimelius

Manuscript writing: All authors

Final approval of manuscript: All authors

\section{REFERENCES}

1. NIH consensus conference: Adjuvant therapy for patients with colon and rectal cancer. JAMA 264:1444-1450, 1990

2. Efficacy of adjuvant fluorouracil and folinic acid in colon cancer: International Multicentre Pooled Analysis of Colon Cancer Trials (IMPACT) investigators. Lancet 345:939-944, 1995

3. Moertel CG, Fleming TR, Macdonald JS, et al: Intergroup study of fluorouracil plus levamisole as adjuvant therapy for stage II/Dukes' B2 colon cancer J Clin Oncol 13:2936-2943, 1995

4. André $T$, Boni $C$, Navarro $M$, et al: Improved overall survival with oxaliplatin, fluorouracil, and leucovorin as adjuvant treatment in stage II or III colon cancer in the MOSAIC trial. J Clin Oncol 27:3109-3116, 2009

5. Yothers G, O'Connell MJ, Allegra CJ, et al: Oxaliplatin as adjuvant therapy for colon cancer: Updated results of NSABP C-07 trial, including survival and subset analyses. J Clin Oncol 29:3768-3774, 2011

6. Schmoll HJ, Tabernero J, Maroun J, et al: Capecitabine plus oxaliplatin compared with fluorouracil/folinic acid as adjuvant therapy for stage III colon cancer: Final results of the NO16968 randomized controlled phase III trial. J Clin Oncol 33:3733-3740, 2015

7. Tournigand $\mathrm{C}$, André T, Bonnetain $\mathrm{F}$, et al: Adjuvant therapy with fluorouracil and oxaliplatin in stage II and elderly patients (between ages 70 and 75 years) with colon cancer: Subgroup analyses of the Multicenter International Study of Oxaliplatin, Fluorouracil, and Leucovorin in the Adjuvant Treatment of Colon Cancer trial. J Clin Oncol 30:3353-3360, 2012

8. Heald RJ, Moran BJ, Ryall RD, et al: Rectal cancer: The Basingstoke experience of total mesorectal excision, 1978-1997. Arch Surg 133:894-899, 1998

9. Påhlman $L$, Bohe $M$, Cedermark $B$, et al: The Swedish rectal cancer registry. Br J Surg 94:1285-1292, 2007

10. Guren MG, Kørner H, Pfeffer F, et al: Nationwide improvement of rectal cancer treatment outcomes in Norway, 1993-2010. Acta Oncol 54:1714-1722, 2015

11. Martling $A L$, Holm $T$, Rutqvist $L E$, et al: Effect of a surgical training programme on outcome of rectal cancer in the County of Stockholm: Stockholm Colorectal Cancer Study Group, Basingstoke Bowel Cancer Research Project. Lancet 356:93-96, 2000

12. Moriya $Y$, Hojo $K$, Sawada $T$, et al: Significance of lateral node dissection for advanced rectal carcinoma at or below the peritoneal reflection. Dis Colon Rectum 32:307-315, 1989

13. Lange MM, Martz JE, Ramdeen $B$, et al: Long-term results of rectal cancer surgery with a systematical operative approach. Ann Surg Oncol 20:1806-1815, 2013

14. Hohenberger W, Weber $K$, Matzel K, et al: Standardized surgery for colonic cancer: Complete mesocolic excision and central ligation-Technical notes and outcome. Colorectal Dis 11:354-364, discussion 364-365, 2009

15. West NP, Kobayashi $H$, Takahashi $K$, et al: Understanding optimal colonic cancer surgery: Comparison of Japanese D3 resection and European complete mesocolic excision with central vascular ligation. J Clin Oncol 30: 1763-1769, 2012

16. Bertelsen CA, Neuenschwander AU, Jansen JE, et al; Danish Colorectal Cancer Group: Disease-free survival after complete mesocolic excision compared with conventional colon cancer surgery: A retrospective, population-based study. Lancet Oncol 16:161-168, 2015

17. Kotake K, Mizuguchi T, Moritani K, et al: Impact of D3 lymph node dissection on survival for patients with T3 and T4 colon cancer. Int J Colorectal Dis 29: 847-852, 2014

18. Shimada $Y$, Hamaguchi $T$, Mizusawa J, et al: Randomised phase III trial of adjuvant chemotherapy with oral uracil and tegafur plus leucovorin versus intravenous fluorouracil and levofolinate in patients with stage III colorectal cancer who have undergone Japanese D2/D3 lymph node dissection: Final results of JCOG0205. Eur J Cancer 50:2231-2240, 2014

19. Bernhoff R, Martling A, Sjövall A, et al: Improved survival after an educational project on colon cancer management in the county of Stockholm: A population based cohort study. Eur J Surg Oncol 41:1479-1484, 2015

20. Colorectal Cancer Collaborative Group: Adjuvant radiotherapy for rectal cancer: A systematic overview of 8,507 patients from 22 randomised trials. Lancet 358:1291-1304, 2001

21. Kapiteijn E, Marijnen CAM, Nagtegaal ID, et al; Dutch Colorectal Cancer Group: Preoperative radiotherapy in combination with total mesorectal excision improves local control in resectable rectal cancer. N Engl J Med 345: 638-646, 2001

22. Sebag-Montefiore D, Stephens RJ, Steele R, et al: Preoperative radiotherapy versus selective postoperative chemoradiotherapy in patients with rectal cancer (MRC CR07 and NCIC-CTG C016): A multicentre, randomised trial. Lancet 373:811-820, 2009

23. Swedish Rectal Cancer Trial: Improved survival with preoperative radiotherapy in resectable rectal cancer. N Engl J Med 336:980-987, 1997

24. Krook JE, Moertel CG, Gunderson LL, et al: Effective surgical adjuvant therapy for high-risk rectal carcinoma. N Engl J Med 324:709-715, 1991

25. Sauer R, Liersch T, Merkel S, et al: Preoperative versus postoperative chemoradiotherapy for locally advanced rectal cancer: Results of the German CAO/ ARO/AIO-94 randomized phase III trial after a median follow-up of 11 years. J Clin Oncol 30:1926-1933, 2012

26. Böckelman $C$, Engelmann BE, Kaprio $T$, et al: Risk of recurrence in patients with colon cancer stage II and III: A systematic review and meta-analysis of recent literature. Acta Oncol 54:5-16, 2015

27. Figueredo A, Coombes ME, Mukherjee S: Adjuvant therapy for completely resected stage II colon cancer. Cochrane Database Syst Rev 3: CD005390, 2008

28. Gill S, Loprinzi CL, Sargent DJ, et al: Pooled analysis of fluorouracil-based adjuvant therapy for stage II and III colon cancer: Who benefits and by how much? $\mathrm{J}$ Clin Oncol 22:1797-1806, 2004

29. Mols F, Beijers T, Lemmens V, et al: Chemotherapy-induced neuropathy and its association with quality of life among 2- to 11-year colorectal cancer survivors: Results from the population-based PROFILES registry. J Clin Oncol 31: 2699-2707, 2013

DOI: $10.1200 / J C O .2015 .65 .3048$; published online ahead of print at www.jco.org on February 22, 2016. 
AUTHORS' DISCLOSURES OF POTENTIAL CONFLICTS OF INTEREST

Should the Benefit of Adjuvant Chemotherapy in Colon Cancer Be Re-Evaluated?

The following represents disclosure information provided by authors of this manuscript. All relationships are considered compensated. Relationships are self-held unless noted. I = Immediate Family Member, Inst = My Institution. Relationships may not relate to the subject matter of this manuscript. For more information about ASCO's conflict of interest policy, please refer to www.asco.org/rwc or jco.ascopubs.org/site/ifc.

Lars A. Påhlman

No relationship to disclose

Werner M. Hohenberger

Stock or Other Ownership: Merck, Bayer HealthCare Pharmaceuticals

Klaus Matzel

Honoraria: Medtronic, Covidien, Cook

Consulting or Advisory Role: Medtronic

\section{Kenichi Sugihara}

Honoraria: Taiho Pharmaceutical, Chugai Pharmaceutical

Research Funding: Chugai Pharmaceutical, Taiho Pharmaceutical

Philip Quirke

Research Funding: Amgen

Travel, Accommodations, Expenses: Amgen

Bengt Glimelius

Honoraria: PledPharma

Research Funding: Swedish Cancer Society 


\section{Acknowledgment}

We dedicate this article to Professor Lars Påhlman (deceased), who played a major role in the improvement in survival from large bowel cancer in Sweden, Europe, and the world. He will be missed by all of us. 